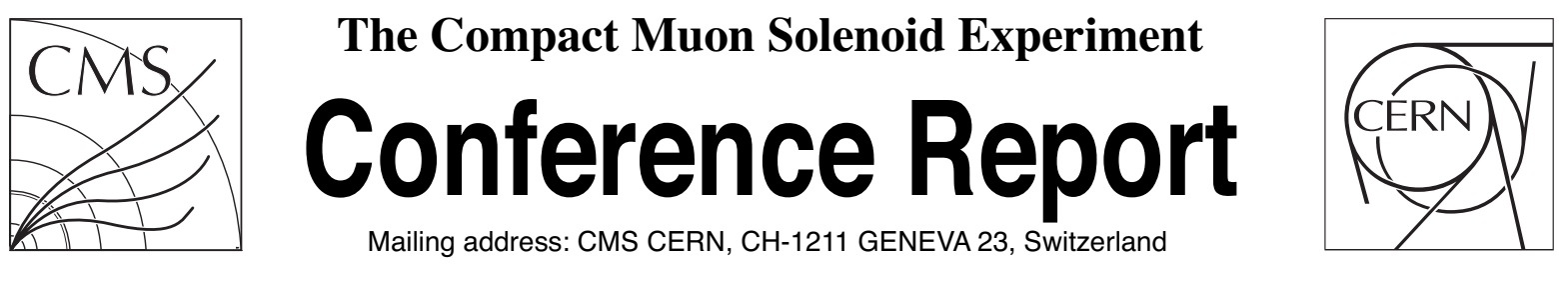

05 November 2014 (v2, 07 November 2014)

\title{
CMS Forward Calorimeters Phase II Upgrade
}

\author{
Burak Bilki for the CMS Collaboration
}

\begin{abstract}
The Phase II Upgrade of the CMS forward calorimeters (electromagnetic and hadronic) originates from the fact that these calorimeters will not be sufficiently performant with the expected High Luminosity LHC conditions, planned to be started in 2025 . The major challenge is to preserve/improve the high performance of the current forward detectors with new devices that can withstand the unprecedented radiation levels and disentangle the very large event pileup. CMS elected two design concepts to be presented in the Phase II Upgrade Technical Proposal Shashlik electromagnetic calorimeter + Hadronic Endcap Rebuild, and High Granularity Calorimeter. The former concept is based on reconstructing the endcap electromagnetic calorimeter with a shashlik design and replacing the active media of the endcap hadron calorimeter with radiation tolerant active media with a possibility to extend the coverage. The latter concept is concentrating on constructing a high granularity (both longitudinally and laterally) calorimeter system with silicon as the active medium for the electromagnetic and the front hadronic sections, and scintillator tiles in the backing hadronic section; all interleaved with absorbers in a sampling calorimeter setup. This report will concentrate on the need for the upgrade, major challenges and the proposed R and D concepts suitable for the Phase II upgrade framework. The existing designs will be discussed with recent information about the beam tests and laboratory measurements.
\end{abstract}




\title{
CMS Forward Calorimeters Phase II Upgrade
}

\author{
Burak Bilki \\ On behalf of the CMS Collaboration
}

\begin{abstract}
The Phase II Upgrade of the CMS forward calorimeters (electromagnetic and hadronic) originates from the fact that these calorimeters will not be sufficiently performant with the expected High Luminosity LHC conditions, planned to be started in 2025. The major challenge is to preserve/improve the high performance of the current forward detectors with new devices that can withstand the unprecedented radiation levels and disentangle the very large event pileup. CMS elected two design concepts to be presented in the Phase II Upgrade Technical Proposal: Shashlik electromagnetic calorimeter + Hadronic Endcap Rebuild, and High Granularity Calorimeter. The former concept is based on reconstructing the endcap electromagnetic calorimeter with a shashlik design and replacing the active media of the endcap hadron calorimeter with radiation tolerant active media with a possibility to extend the coverage. The latter concept is concentrating on constructing a high granularity (both longitudinally and laterally) calorimeter system with silicon as the active medium for the electromagnetic and the front hadronic sections, and scintillator tiles in the backing hadronic section; all interleaved with absorbers in a sampling calorimeter setup. Here we concentrate on the need for the upgrade, major challenges and the proposed R\&D concepts suitable for the Phase II upgrade framework with recent information about the beam tests and laboratory measurements.
\end{abstract}

\section{INTRODUCTION}

The Compact Muon Solenoid (CMS) [1] is a generalpurpose detector designed to run at the highest luminosity provided by the CERN Large Hadron Collider (LHC). The CMS detector calorimeter has been designed to detect cleanly the diverse signatures of new physics through the measurement of jets with moderate precision and by measuring missing transverse energy flow. The CMS experiment has a $4 \mathrm{~T}$ superconducting solenoidal magnet of length $13 \mathrm{~m}$ and inner diameter $5.9 \mathrm{~m}$. The magnet determines many of the features of the CMS calorimeters because the barrel and end-cap calorimeters are located inside this magnet.

The CMS ECAL (electromagnetic calorimeter) is a homogeneous crystal calorimeter and consists of a barrel (EB) section and two endcaps (EE) made in total of $\sim 76 \mathrm{kPWO}_{4}$ crystals, with a $3 \mathrm{X}_{0}$ lead - silicon strip preshower (ES) detector in the endcap section. The scintillation light is read-out by a pair of avalanche photodiodes (APDs) for each EB crystal and a vacuum phototriode for each EE crystal. Further information about the ECAL can be found in [2], [3], [4], [5], [6].

The HCAL (hadron calorimeter) is used to measure the energy of hadronic showers, as well as their angle and position,

Manuscript received November 4, 2007.

Burak Bilki is with University of Iowa, Iowa City, IA 52242 USA and Argonne National Laboratory, Argonne, IL 60439 USA (e-mail: burakbilki@uiowa.edu). needed for the generation of calorimeter trigger, and offline reconstruction of jets and missing transverse energy. The CMS HCAL contains 9072 readout channels organized into four subsystems: barrel (HB, 2592 channels), endcap (HE, 2592 channels), outer (HO, 2160 channels) and forward (HF, 1728 channels). The performance of the HB, HE, HO, and HF were also extensively investigated and are reported in [2], [6], [7], [8], [9].

Currently, the LHC is in the Long Shutdown 1 (LS1) period and the CMS along with the LHC and other experiments is going through various upgrade processes. The start-up in 2015 , Run 2, is aimed at collision energy of $13 \mathrm{TeV}$ and an instantaneous luminosity at the nominal value i.e. $1 \times 10^{34}$ $\mathrm{cm}^{-2} \mathrm{~s}^{-1}$. The performance reach is specified in terms of levelled luminosity. This implies that the peak luminosity that is virtually required to reach such a leveled luminosity is much higher. More information about the levelling approaches can be found in [13].

At the end of Run 2, LHC is planned to reach the collision energy of $14 \mathrm{TeV}$ and instantaneous luminosity of $1.6 \times 10^{34}$ $\mathrm{cm}^{-2} \mathrm{~s}^{-1}$. Nominal bunch spacing of $25 \mathrm{~ns}$ will be used for this run period on. The expected average pileup will be around 40. The target integrated luminosity before Long Shutdown 2 (LS2) is $\sim 200 \mathrm{fb}^{-1}$.

During Run 3, which will start after LS2 in 2020, LHC will reach $2 \mathrm{x}$ nominal luminosity, again in a levelled approach. The collision energy and the bunch spacing parameters will be at their nominal values, $14 \mathrm{TeV}$ and $25 \mathrm{~ns}$ respectively. The projected average pileup will be $\sim 50$. Run 3 is aimed at an integrated luminosity of $500 \mathrm{fb}^{-1}$.

Long Shutdown 3 (LS3) between 2023-2025 is the planned time interval for the significant detector upgrades necessary for the operations beyond 2025. Following LS3, in Run 4, the LHC will operate in the High-Luminosity LHC (HL-LHC) regime. This will be realized by instantaneous luminosities between 5 to 10 times the nominal luminosity at the nominal collision energy and bunch spacing. The target integrated luminosity in the HL-LHC era is $\sim 3000 \mathrm{fb}^{-1}$.

Figure 1 summarizes the LHC schedule as of today.

Here we report on the necessity of CMS forward detector upgrades for the High Luminosity LHC (HL-LHC) era and describes the possible upgrade options considered by CMS. Here, the forward region is considered to be $1.4<|\eta|<5$, where $\eta$ is the pseudorapidity. The upgrade detectors described are planned to be installed during LS3. 


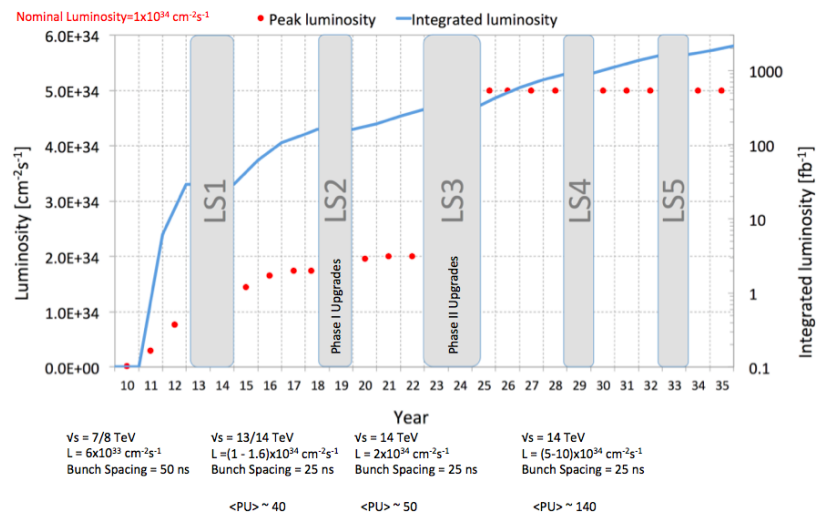

Fig. 1. LHC schedule as of today. Also indicated are the target integrated luminosities per run period and expected average number of pile up events. HL-LHC era is following LS3.

\section{Performance Projections of the CMS Forward CALORIMETERS TOWARDS HL-LHC}

The ECAL Endcap detectors, covering $1.4<|\eta|<3$, will experience progressive deterioration of energy resolution and trigger efficiency with strong dependence on the pseudorapidity. Figure 2 (top) shows the fraction of the ECAL response at various operating conditions to the nominal response of the ECAL as a function of pseudorapidity. The EE detectors should be replaced for optimal operation in the HL-LHC era.

Figure 2 (middle) shows the response degradation of the Hadron Endcap calorimeters for different conditions and as a function of the pseudorapidity. HE covers $1.3<|\eta|<3$ and L1 and L7 represent the longitudinal samplings at 1 and 7 layer depths. The green solid and dashed lines are based on the data acquired between 2010 and 2013, $500 \mathrm{fb}^{-1}$ and $3000 \mathrm{fb}^{-1}$ lines (blue and red, solid and dashed) are predictions based on the measurements. The performance degradation dictates that the HE detectors will need to be upgraded/replaced for the HL-LHC running conditions.

The coverage between $3<|\eta|<5$ is provided by the Hadron Forward (HF) calorimeters. Figure 2 (bottom) shows the signal loss in HF at three different pseudorapidity ranges as a function of the integrated luminosity. The data points are the mesurements with the laser calibration system in 20112012 and the solid lines are the predictions based on these measurements. The predictions do not take into account the self-curing of the quartz fibers during the shutdown periods. The HF calorimeters will be sufficiently performant through HL-LHC and there is no upgrade planned by CMS.

\section{CONCEPTS CONSIDERED For Phase II UpgRade}

There are two options considered by CMS for the Phase II Detector Upgrade: Shashlik electromagnetic calorimeter + HE rebuild; High Granularity Calorimeter.

\section{A. Shashlik EE + HE Rebuild}

This option is based on replacing the EE + ES with the shashlik design ECAL and replacing the active media of the $\mathrm{HE}$ and extending the longitudinal and transverse segmentation of the calorimeter.
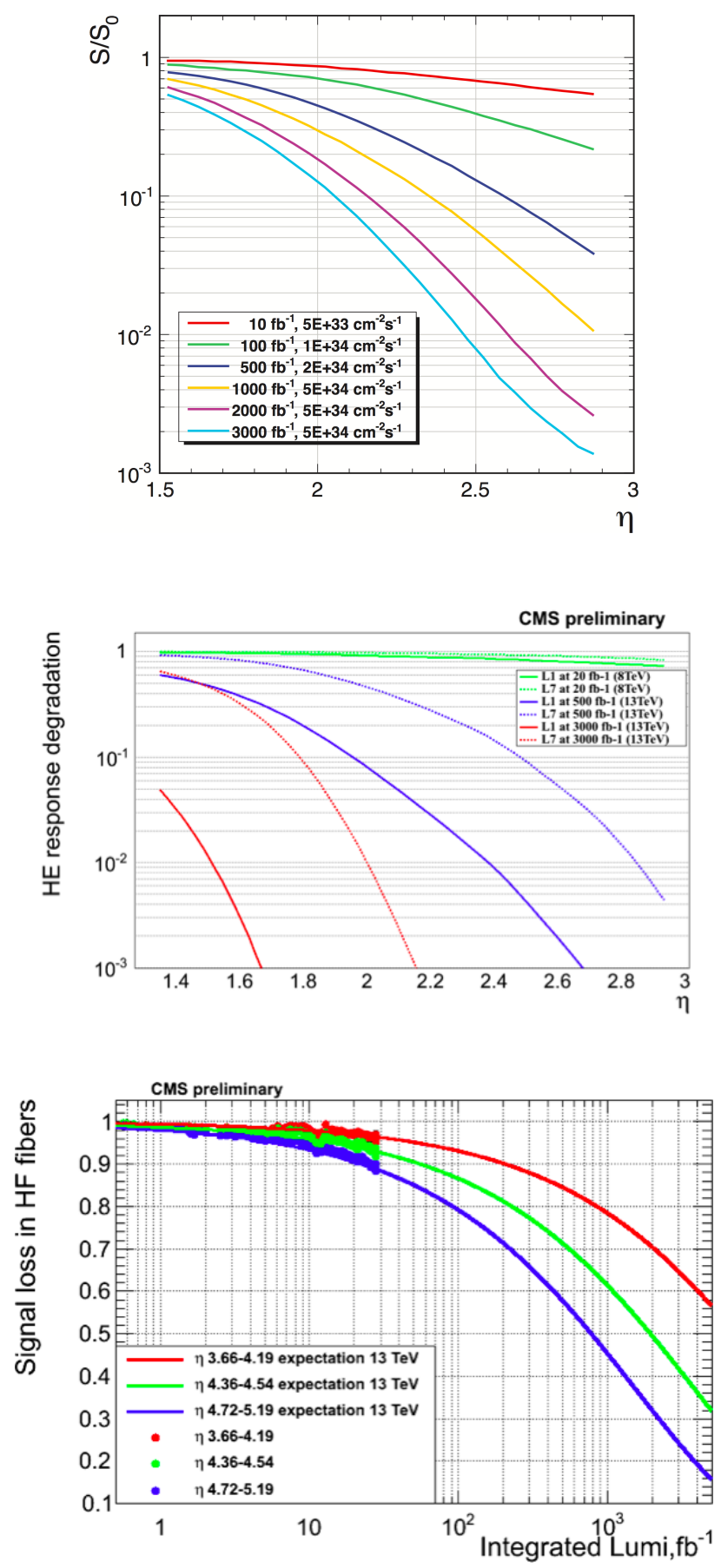

Fig. 2. Simulated fraction of ECAL response to $50 \mathrm{GeV}$ electrons under different operating conditions as a function of pseudorapidity (top), response degradation of the Hadron Endcap calorimeters at different operating points for two different longitudinal segmentations in the calorimeter and as a function of pseudorapidity (middle) and the signal loss in Hadron Forward calorimeters at three different pseudorapidity ranges as a function of the integrated luminosity (bottom). 
The expected performance of various EE replacement detectors has been studied for long and of these, an architecture based upon W absorber layers and LYSO sampling layers read out with quartz capillaries with waveshifter cores and GaInP photosensors has been found to potentially provide the best electromagnetic energy resolution $\sim 10 \% / \sqrt{E}$ in the absence of radiation damage and pileup.

Figure 3 shows the preliminary design of the basic shashlik module. A single module consists of $282.5 \mathrm{~mm}$ thick W plates and $291.5 \mathrm{~mm}$ thick LYSO crystals both having $14 \mathrm{~mm}$ lateral sizes. The length of a single module is $114 \mathrm{~mm}$. The readout is provided by 4 wavelength shifting quartz capillaries, which are coupled to 1 or $2 \mathrm{GaInP}$ photodetectors. The module also consists of a calibration fiber running through the center. The shashlik modules have shorter length and smaller transverse size resulting in a larger number of modules. The modules also have smaller Moliere radius and radiation length. There are also substantial differences in terms of the light yield and temperature dependence. Table I shows the comparison of the parameters of shashlik EE and $\mathrm{PbWO}_{4} \mathrm{EE}$.

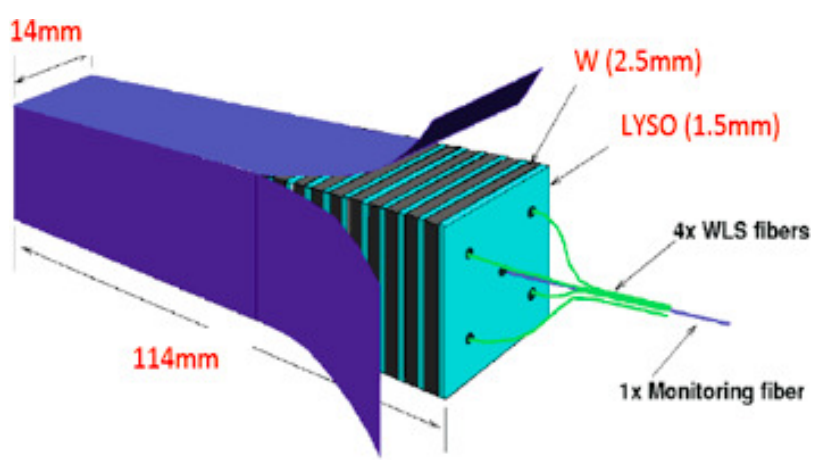

Fig. 3. Design of the basic shashlik module.

TABLE I

PARAMETRIC COMPARISON OF THE SHASHLIK EE AND $\mathrm{PBWO}_{4}$ EE.

\begin{tabular}{|l|c|c|}
\hline & W/LYSO(Ce) & PbWO $_{4}$ \\
\hline Length (mm) & 114 & 220 \\
\hline Transverse Size (mm) & 14 & 28.6 \\
\hline Detector Modules & 60,800 & 14,648 \\
\hline Moliere Radius (mm) & 13.7 & 21 \\
\hline Radiation Length - $\mathbf{X}_{0}$ (mm) & 5.1 & 8.9 \\
\hline Light Yield (Relative to NaI) & 85 & 0.3 \\
\hline Emission Wavelength (nm) & 420 & 420 \\
\hline Decay Time (ns) & 40 & 25 \\
\hline Light Output (pe/MeV) & 6 & 4.5 \\
\hline Temperature Dependence (\%/C) & -0.2 & -2.2 \\
\hline
\end{tabular}

The major advantages of LYSO are brightness and density. Stability of the scintillation mechanism and material costs are the outstanding $\mathrm{R} \& \mathrm{D}$ issues. Other crystals, in particular $\mathrm{CeF}_{3}$, are being investigated. The merits of $\mathrm{CeF}_{3}$ include its lighter components that lead to less hadronic radiation damage at a given dose and recovery from hadronic radiation damage (unlike LYSO in which hadronic radiation damage is cumulative). In addition, the scintillation time constant is shorter (below $30 \mathrm{~ns}$ versus $40 \mathrm{~ns}$ for LYSO); the peak emission is in the ultraviolet $(310-340 \mathrm{~nm}$ versus $430 \mathrm{~nm}$ for
LYSO) and so will require a different wavelength shifter that emits in the 400 to $500 \mathrm{~nm}$ range. Finally, $\mathrm{CeF}_{3}$ is relatively more insensitive to temperature compared to LYSO.

The current options for wavelength shifting capillaries are thick quartz wall and quartz rod. The thick quartz wall capillary has an outer diameter of $1.2 \mathrm{~mm}$ and an inner diameter of $0.4 \mathrm{~mm}$. The core is a liquid wavelength shifter with the ends plugged. Photodetector is coupled to the quartz annulus and the far end is mirrored. The quartz rod has $1 \mathrm{~mm}$ diameter with powdered wavelength shifter on the outer surface as a thin layer. The wavelength shifter for both cases is envisaged as J2 (Y11) or DSB1 with other alternatives possible. The major advantage of the quartz capillaries is radiation hardness of both quartz and the wavelength shifter. The $R \& D$ requirements are the irradiation tests and stability studies of various options and test beam measurements. Cerium-doped quartz fibers are also considered among alternatives.

Figure 4 shows the sketches of thick quartz wall (top left) and quartz rod (top right) wavelength shifting capillaries. Figure 4 bottom shows the study of light collection from capillaries as a function of gamma irradiation. The measurements were performed at three irradiation levels (0, 50 and $150 \mathrm{Mrad})$ for two different cases: unblocked and blocked. In the latter case, the core of the thick quartz wall capillary is blocked by fusili wire. The effect of blocking is clearly visible as a flattened response along the capillary.

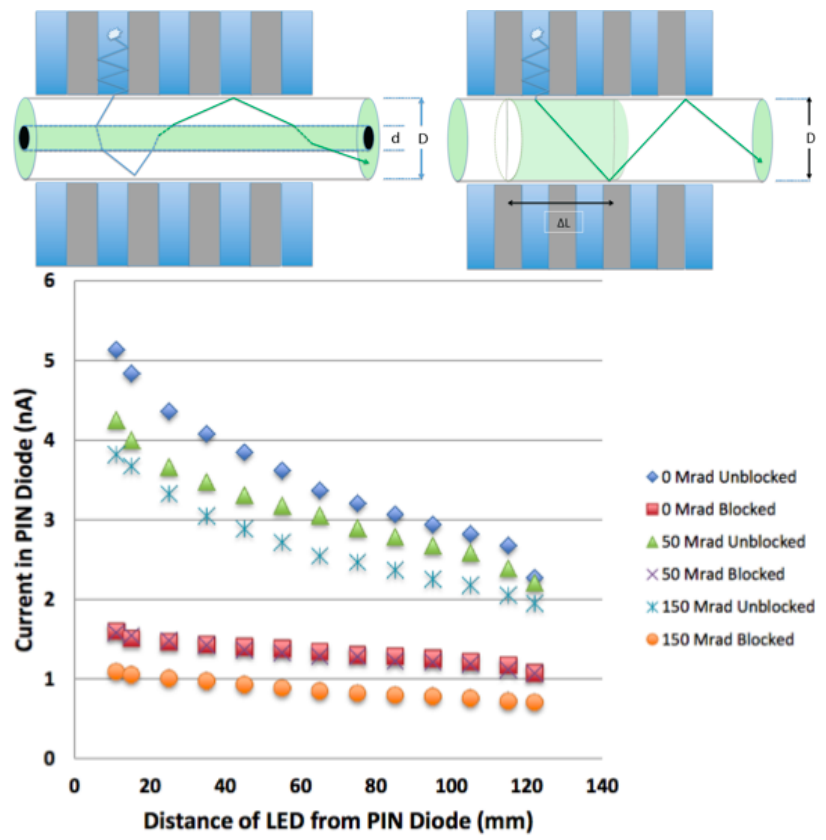

Fig. 4. Possible designs of wavelength shifting capillaries: Thick quartz wall (top left) and quartz rod (top right). Study of light collection from thick quartz wall capillaries as a function of gamma irradiation (bottom).

It has been shown that photodetectors with similar performance to SiPMs with Ga compounds, specifically GaAs, can be fabricated. The development of photosensors from GaInP that have the potential of being 200,000 times as radiation hard as $\mathrm{Si}$ based photosensors is underway. The fabrication of SPADs (single photon avalanche devices) made 
from GaInP is completed and the SPADs achieved the expected low dark currents of $1 \mathrm{pA}$. It is worth pointing out that GaInP is not considered to be an "exotic" material in the semiconductor industry and indeed, is used along with GaAs in many applications. It now appears that this will be the leading contender for the shashlik EE.

The proposed readout scheme for EE is built upon existing strategies i.e. this can be made compatible with the readout scheme foreseen for the ECAL barrel at Phase II or with the QIE10 electronics aimed for HE at Phase I.

The two potential roles of HE rebuild in the Phase II upgrade are the fully functioning hadron calorimeter behind shashlik EE and the backing hadron calorimeter of the HGC (high granularity calorimeter). In this context and in the view of the HL-LHC conditions, the proposed R\&D paths for the HE rebuild can be summarized as follows:

- Study of radiation damage to the CMS HE and of possible alternative solutions

- Radiation study of present system

- Development and radiation damage study of finger tiles, liquid scintillator tiles and green emitting scintillators

- Finger scintillator option for HE upgrade beyond Phase I

- Understanding of radiation damage of present tiles at $30 \mathrm{fb}^{-1}$

- Radiation damage of present tiles up to $7 \mathrm{Mrad}(700$ $\mathrm{fb}^{-1}$ )

- Radiation damage of finger tiles up to $25 \mathrm{Mrad}(3000$ $\mathrm{fb}^{-1}$ )

- Crystal fiber R\&D

- Layer of LuAG crystal fibers coupled to quartz capillaries.

- Development of radiation-hard WLS Films, Tiles, and Fibers

- Quartz plate R\&D

- Development of UV-absorbing WLS fibers

There has been quite a lot of progress both in the laboratories and the test beams recently in terms of understanding the damage of the current HE detectors and investigating possible alternatives. As an example, Fig. 5 shows the radiation damage of the HE scintillators measured during CMS data taking in 2012 at two different depth segmentations (red and blue points) also the degredation of the same type of scintillators with various sizes when exposed to Co-60 source (hollow points and dashed lines). The dependence of the degredation on the tile size is visible in the laboratory measurements. The signal loss observed in the CMS happens at much lower doses than in the laboratory measurments. This effect is attributed to the difference in the dose rate (Mrad/hr) besides the total integrated dose.

While following the HE rebuild $R \& D$ paths, the mechanical design will also be studied to enable larger longitudinal and lateral coverage.

\section{B. High Granularity Calorimeter (HGC)}

A high granularity calorimeter with a detailed sampling in both the hadronic and the electromagnetic sections with ex-

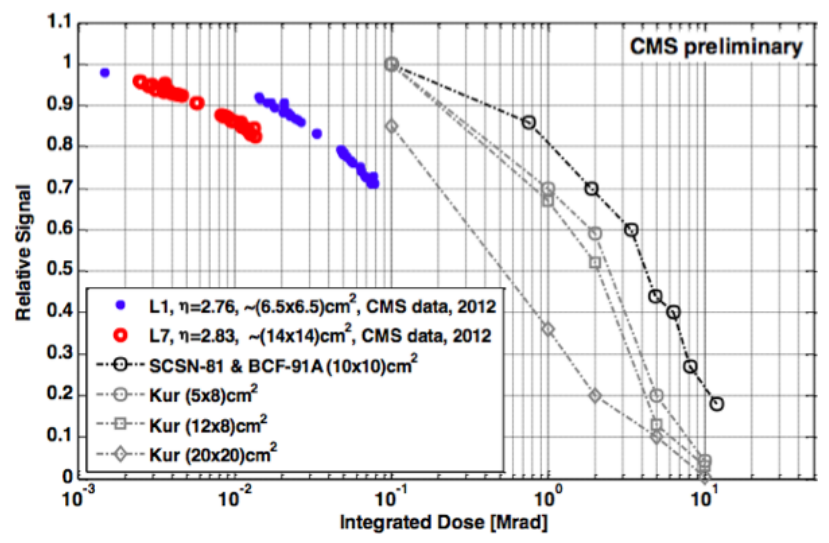

Fig. 5. Response degredation of the HE scintillator tiles as a function of integrated dose, both CMS and laboratory measurements.

cellent pointing capability could have significant performance benefits. The approach that has been developed is a sampling calorimeter with layers of silicon detectors that feature very high longitudinal and lateral granularities in the electromagnetic and the front hadronic calorimeter sections, and a coarser segmentation backing hadronic calorimeter section which is under the development framework of the HE rebuild option.

The major components of the HGC are summarized below:

- Electromagnetic Calorimeter:

- 30 samplings of lead/copper total of $25 \mathrm{X}_{0}$

* 10 layers of $0.5 \mathrm{X}_{0} / 10$ layers of $0.8 \mathrm{X}_{0} / 10$ layers of $1.2 \mathrm{X}_{0}$

* Pad size $0.9 \mathrm{~cm}^{2}$ for first 20 layers, $1.8 \mathrm{~cm}^{2}$ for the last 10 layers

- $420 \mathrm{~m}^{2}$ of silicon pad detectors

- 3.7M channels

- Front Hadronic Calorimeter:

- 4 interaction lengths

- 12 layers of brass/silicon each 0.33 interaction lengths

- Pad size is $1.8 \mathrm{~cm}^{2}$

- $1.4 \mathrm{M}$ channels

- Backing Calorimeter:

- Five interaction lengths (e.g. sampling of 0.5 interaction lengths)

- Since the radiation levels are lower, plastic scintillators can be used as active medium

The HGC is envisaged to have an unprecedented granularity resulting in $5.1 \mathrm{M}$ readout channels utilizing $670 \mathrm{~m}^{2}$ of Silicon as the active medium. Table II shows the parameters of the electromagnetic and front hadron calorimeter sections of the HGC.

All Silicon sensors will be made with the standard $320 \mu \mathrm{m}$ thick wafers. For regions of low radiation, $300 \mu \mathrm{m}$ depletion depth is foreseen. The medium and high radiation regions will be equipped with $200 \mu \mathrm{m}$ and $100 \mu \mathrm{m}$ depletion length sensors respectively. The wafers will be hexagonal shape and they will be placed in a cassette structure with integrated cooling pipes. Figure 6 shows the sketch of the cassette (left) and a possible placement in the wedges (right). 
TABLE II

PARAMETERS OF THE ELECTROMAGNETIC (EE) AND FRONT HADRON (FH) CALORIMETER SECTIONS OF HGC.

\begin{tabular}{|l|c|c|c|}
\hline & EE & FH & Total \\
\hline Area of Silicon $\left.\mathbf{( m}^{2}\right)$ & 420 & 250 & 670 \\
\hline Channels & $3.7 \mathrm{M}$ & $1.4 \mathrm{M}$ & $5.1 \mathrm{M}$ \\
\hline Detector Modules & $19 \mathrm{~K}$ & $11 \mathrm{~K}$ & $30 \mathrm{~K}$ \\
\hline Weight One Endcap (tonnes) & 16 & 63 & 79 \\
\hline Number of Plates & 30 & 12 & 42 \\
\hline Front-end Power (kW) & $70-80$ & $20-30$ & $90-110$ \\
\hline
\end{tabular}
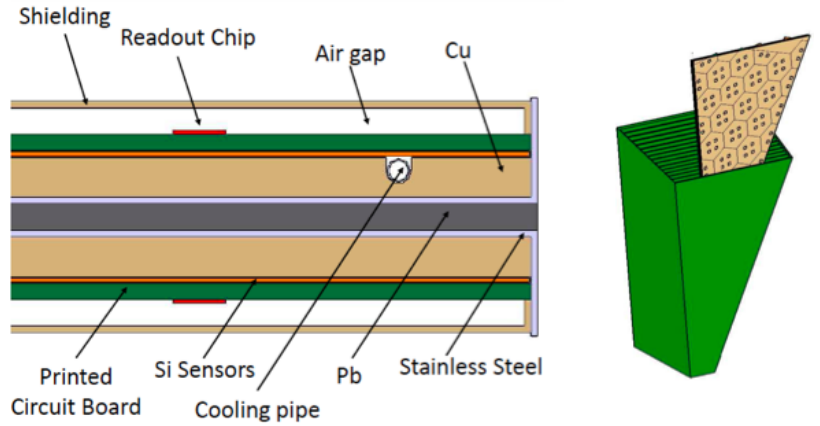

Fig. 6. The HGC cassette to house Silicon wafers, associated front-end electronics and the cooling pipes (left) and a possible placement in the endcap wedges (right).

The front-end electronics design for HGC utilizes an input capacitor of $50-100 \mathrm{pF}$, a time constant of the order of 15 $20 \mathrm{~ns}$ and a single MIP response larger than $15 \mathrm{k}$ e- before damage. On-chip digitization for each channel will be at 40 MHz. The sum of 4 (2) adjacent pads will serve as the Level 1 Trigger primitives with 8-10 bit resolution. The target power consumption per channel is $15 \mathrm{~mW}$. The sketch of the frontend electronics design for HGC is shown in Fig. 7.

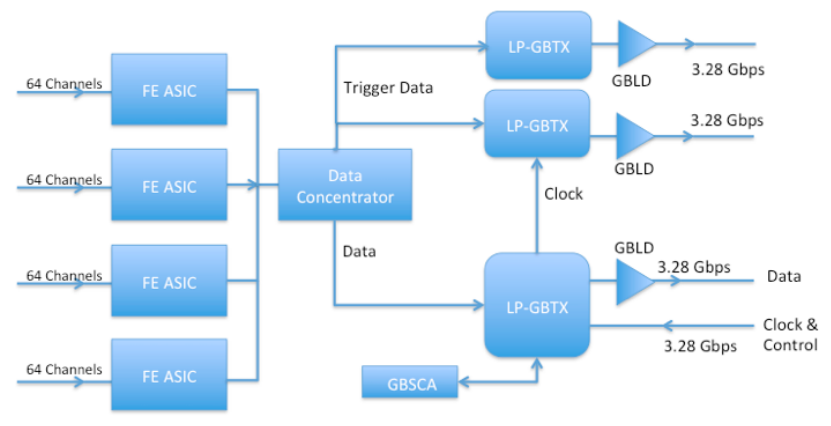

Fig. 7. The sketch of the front-end electronics design for HGC.

\section{BEAM TESTS}

The CMS Forward Calorimetry Upgrade groups have already started the test beam effort in December 2013 at Fermilab Test Beam Facility (FTBF) [14]. The groups perform their tests under the collaboration of Fermilab T-1041 "CMS Forward Calorimetry R\&D" Experiment [15]. In an extended effort, the upgrade groups are currently performing beam tests at CERN H2 and H4 beam lines [16] and are planning irradiation tests at CERN and other non-HEP facilities in the near future.

\section{SUMmary}

The CMS endcap electromagnetic and hadron calorimeters will experience significant performance degradation due to high radiation environment exposed by the High Luminosity LHC conditions and will need to be replaced/upgraded during Long Shutdown 3. In this context, CMS considers two upgrade scenarios:

- Shashlik EE + HE Rebuild: The endcap electromagnetic calorimeter as well as the endcap preshower detector will be replaced by the shashlik type electromagnetic calorimeter, and the hadron endcap calorimeter will be improved in terms of active media and lateral and longitudinal coverage.

- High Granularity Calorimeter: A high granularity calorimeter based on Silicon as the active medium will be constructed in two sections with varying segmentation electromagnetic and front hadronic. The backing hadron calorimeter with a coarser segmentation will be built with the HE rebuild options.

Both scenarios have rigorous $\mathrm{R} \& \mathrm{D}$ plans on active media (e.g. scintillators, silicon sensors, crystals), readout components (e.g. fibers and photodetectors) and readout electronics (e.g. on-detector electronics). Most of the R\&D project groups have recently completed the preliminary phases of beam tests, and are working extensively on the engineering designs and various implementation details.

\section{REFERENCES}

[1] S. Chatrchyan et al. (CMS collabration), JINST 3 S08004, 2008.

[2] G. L. Bayatian et al. (CMS collabration),CERN/LHCC-2006-001, 2006.

[3] P. Adzic et al. (CMS ECAL Collaboration), Eur. Phys. J. C 44 1-10, 2006.

[4] P. Adzic et al. (CMS ECAL Collaboration), JINST 2 P04004, 2007.

[5] S. Chatrchyan et al. (CMS Collaboration), JINST 8 S09009, 2013.

[6] S. Abdullin et al. (CMS ECAL and HCAL Collaborations), Eur. Phys. J. C 60 359-373, 2009.

[7] S. Abdullin et al. (CMS HCAL Collaboration), Eur. Phys. J. C 55159 171, 2008.

[8] S. Abdullin et al. (CMS HCAL Collaboration), Eur. Phys. J. C 53139 166, 2008.

[9] G.L. Bayatian et al. (CMS Collaboration), CMS-NOTE-2008/010, 2008.

[10] G. Aad G et al. (ATLAS Collaboration), Phys. Lett. B 716 1-29, 2012.

[11] S. Chatrchyan et al. (CMS Collaboration), Phys. Lett. B 716 30-61, 2012.

[12] R. Alemany-Fernandez et al., CERN-ACC-NOTE-2013-0041, 2013.

[13] M. Giovannozzi, AIP Conference Proceedings 1560 686, 2013.

[14] http://www-ppd.fnal.gov/FTBF/

[15] http://www-ppd.fnal.gov/FTBF/TSW/PDF/T1041_tsw_signed.pdf

[16] http://sba.web.cern.ch/sba/BeamsAndAreas/resultbeam.asp?beamline $=\mathrm{H} 2$, http://sba.web.cern.ch/sba/BeamsAndAreas/resultbeam.asp?beamline=H4 